\title{
ECG-derived Respiratory Rate in Atrial Fibrillation
}

\author{
Spyridon Kontaxis, Jesús Lázaro, Member IEEE, Valentina D. A. Corino, Frida Sandberg, \\ Raquel Bailón, Pablo Laguna, Fellow IEEE, and Leif Sörnmo, Fellow IEEE
}

\begin{abstract}
Objective: The present study addresses the problem of estimating the respiratory rate from the morphological ECG variations in the presence of atrial fibrillatory waves (f-waves). The significance of performing f-wave suppression before respiratory rate estimation is investigated. Methods: The performance of a novel approach to ECG-derived respiration, named "slope range" (SR) and designed particularly for operation in atrial fibrillation (AF), is compared to that of two well-known methods based on either R-wave angle (RA) or QRS loop rotation angle (LA). A novel rule is proposed for spectral peak selection in respiratory rate estimation. The suppression of $f$-waves is accomplished using signal- and noise-dependent QRS weighted averaging. The performance evaluation embraces real as well as simulated ECG signals acquired from patients with persistent AF; the estimation error of the respiratory rate is determined for both types of signals. Results: Using real ECG signals and reference respiratory signals, rate estimation without f-wave suppression resulted in a median error of $0.015 \pm 0.021 \mathrm{~Hz}$ and $0.019 \pm 0.025 \mathrm{~Hz}$ for $\mathrm{SR}$ and RA, respectively, whereas LA with f-wave suppression resulted in $0.034 \pm 0.039 \mathrm{~Hz}$. Using simulated signals, the results also demonstrate that f-wave suppression is superfluous for SR and RA, whereas it is essential for LA. Conclusion: The results show that SR offers the best performance as well as computational simplicity since f-wave suppression is not needed. Significance: The respiratory rate can be robustly estimated from the ECG in the presence of AF.
\end{abstract}

\section{INTRODUCTION}

$\mathbf{R}$ ESPIRATORY dysfunction is associated with atrial fibrillation (AF) since reduced lung function, decreased oxygenation, hypercapnia, pulmonary hypertension, chronic obstructive pulmonary disease, and sleep-disordered breathing (SDB) have been identified as independent predictors of AF [1]-[4]. Alterations in respiratory physiology due to a progressive decline in lung function may provoke alterations in cardiac structure and function through changes in atrial

This work was funded under the project RTI2018-097723-B-I00 (Reference Group BSICoS T39-17R) by FEDER 2014-2020, Gobierno de Aragón, European Social Fund, and European Union's Framework Programme for Research and Innovation Horizon 2020 (2014-2020) under the Marie Skłodowska-Curie Grant Agreement No. 745755. This work was supported by CIBER which is a center of the Instituto de Salud Carlos III in assistance from the European Regional Development Fund. The computation was performed by the ICTS "NANBIOSIS", more specifically by the High Performance Computing Unit of the CIBER in Bioengineering, Biomaterials \& Nanomedicine (CIBER$\mathrm{BBN}$ ) at the University of Zaragoza. Asterisk indicates corresponding author

* S. Kontaxis, J. Lázaro, R. Bailón, and P. Laguna are with the Biomedical Signal Interpretation and Computational Simulation (BSICoS) group at the Aragón Institute of Engineering Research (I3A), IIS Aragón, University of Zaragoza, Spain, (e-mail: sikontax@unizar.es)

J. Lázaro, R. Bailón, and P. Laguna, are with CIBER de Bioingeniería, Biomateriales y Nanomedicina (CIBER-BBN), Spain.

J. Lázaro is with the Department of Biomedical Engineering, University of Connecticut, Storrs, CT 06269, USA.

V. D. A. Corino is with the Department of Bioengineering, Politecnico di Milano, 20133 Milan, Italy.

F. Sandberg and L. Sörnmo are with the Department of Biomedical Engineering and the Center of Integrative Electrocardiology, Lund University. electrophysiology, leading to increased incidence of cardiac arrhythmia [5].

A variety of pathophysiological mechanisms, including apnea-induced hypoxia and intrathoracic pressure shifts, have been associated with AF in patients with SDB [6], where the risk of new AF episodes is markedly increased shortly after apneas and hypopneas during sleep [7], [8]. Moreover, patients with SDB may be predisposed to arrhythmias because of an increased sympathetic tone. Autonomic imbalance could alter atrial electrophysiology and provoke AF [9]. These clinical observations exemplify the complex interplay between the respiratory, cardiovascular, and autonomic nervous systems, and highlight the need for tools to monitor respiration in patients with AF [10]. In clinical practice, respiratory rate is currently measured using techniques which either interfere with normal breathing (nasal thermistors, carbon dioxide sensors) cause discomfort (transthoracic inductance and plethysmography), or do not offer acceptable quality (impedance pneumography) [11], [12]. Since these techniques are impractical in monitoring, the ECG can be used to indirectly estimate a respiratory signal, known as the ECG-derived respiration (EDR) signal.

The majority of published EDR methods explore beat morphology or heart rate information [13], [14]. For example, the respiratory rate was obtained as the dominant frequency of the estimated rotation angles of the electrical axis of the heart, accomplished by spatiotemporal alignment of successive vectorcardiographic loops of the QRS complex relative to a reference loop [15], [16]. The QRS amplitude is another characteristic which has been explored, especially in the context of SDB monitoring [17], [18]. Principal component analysis (PCA) and kernel PCA have been used for extracting the respiratory rate from beat-to-beat changes in morphology [19][21]. The up- or downslope of the QRS complex [22] and the amplitude difference between the $R$ and the $S$ waves [23] are other characteristics which reflect respiration-induced changes.

The above-mentioned EDR methods have explored the idea of retrieving respiratory information from the ECG during sinus rhythm, whereas EDR methods in arrhythmia remains largely unexplored. One of the very few EDR studies involving AF patients reported on low detection sensitivity of SDB [24]. The performance of techniques exploring morphology may deteriorate due to the presence of atrial fibrillatory waves (fwaves) which can mask the respiratory information. Therefore, f-wave suppression may have to be performed before respiratory rate estimation. It should be noted that methods exploring heart rate information cannot be used in AF since the rhythm is not controlled by the autonomic nervous system and thus, respiratory sinus arrhythmia is not present.

The novelty of the present study consists in the estimation of respiratory rate in the presence of f-waves, using either the 
QRS loop rotation angle (LA) [16], the R-wave angle (RA) [22], which have been reported to be robust in noisy situations, or the novel slope range (SR) approach to computing the EDR power spectrum. The performance is also compared to the QRS area (A) method, originally proposed in [25] and used in the SDB study presented in [24]. Moreover, a novel technique for selecting the spectral peak related to respiration is proposed, being particularly robust to spurious spectral components caused by f-waves.

Performance is studied on a database of AF patients consisting of simultaneously recorded ECG and respiration signals as well as on simulated signals, accounting for different morphologies, rhythms, induced respiratory modulation, fwaves, and the presence of noise at different signal-to-noise ratios (SNRs).

The paper is organized as follows. Section II describes the signal processing involved with the rate estimation. The data sets and the setup of the performance evaluation are described in Section III and IV, respectively. Section V presents the results, which are then subject to discussion in Section VI.

\section{Methods}

\section{A. Preprocessing}

The ECG is subjected to band-pass filtering in the band $[0.5,45] \mathrm{Hz}$, and, the beats for each lead are detected. The length of the QRS interval is set to $140 \mathrm{~ms}$, starting $110 \mathrm{~ms}$ before the end of the QRS complex and ending $30 \mathrm{~ms}$ after.

1) Noisy beat identification: Noisy beats are identified by the method in [16] and discarded from further analysis. Moreover, beats whose cross-correlation coefficient to a reference QRS is lower than 0.8 are discarded; thus, only beats with dominant morphology are analyzed. The reference QRS is taken as the average of the first 10 beats of the recording having similar morphology.

2) Suppression of $f$-waves: In contrast to AF signal processing, where the objective is to extract and analyze the fwaves, the objective is here to suppress the f-waves, while preserving respiratory related QRS variability, before respiratory rate estimation is performed. Signal- and noise-dependent weighted QRS averaging is used for this objective [26], see also [27], being based on the same principle as average beat subtraction (ABS). The $i$ :th observed beat $x_{i}(n)$ is modeled as:

$$
x_{i}(n)=x_{\mathrm{QRS}, i}(n)+x_{f, i}(n), \quad i=1, \ldots, M,
$$

where $x_{\mathrm{QRS}, i}(n)$ and $x_{f, i}(n)$ are the QRS complex and the f-waves, respectively, and $M$ is the number of beats with dominant morphology. The QRS complex is characterized by the time-dependent variance $\sigma_{\mathrm{QRS}}^{2}(n)$ and the f-waves by the constant variance $\sigma_{f}^{2}$. The f-wave suppressed QRS complex of the $i$ :th beat is obtained as a linear combination of $M$ beats:

$$
\hat{x}_{\mathrm{QRS}, i}(n)=\sum_{m=1}^{M} w_{i, m}(n) x_{m}(n),
$$

where the weights $w_{i, m}(n)$ are given by [27]:

$$
w_{i, m}(n)=\frac{1}{M} \frac{\sigma_{f}^{2}}{\sigma_{f}^{2}+\sigma_{\mathrm{QRS}}^{2}(n)}+\frac{\sigma_{\mathrm{QRS}}^{2}(n)}{\sigma_{f}^{2}+\sigma_{\mathrm{QRS}}^{2}(n)} \delta(i-m) .
$$

The f-wave variance $\sigma_{f}^{2}$ is estimated from the observed signal by computing the sample variance of the concatenated TQ intervals of all beats, following bandpass filtering in the band $[0.5,30] \mathrm{Hz}$ to preserve f-wave related frequency components and reduce the influence of noise [27]. The QRS variance $\sigma_{\mathrm{QRS}}^{2}(n)$ is estimated by

$$
\hat{\sigma}_{\mathrm{QRS}}^{2}(n)=\max \left\{\frac{1}{M-1} \sum_{m=1}^{M}\left(x_{m}(n)-\bar{x}(n)\right)^{2}-\sigma_{f}^{2}, 0\right\},
$$

where $\bar{x}(n)$ denotes the ensemble average of $x_{1}(n), \ldots, x_{\mathrm{M}}(n)$.

This approach uses weights $w_{i, m}(n)$ which not only change from sample-to-sample within each beat given the timedependent $\mathrm{QRS}$ variance $\sigma_{\mathrm{QRS}}^{2}(n)$, but also from beat-to-beat since more weight is assigned to the $i$ :th beat $(m=i)$, thereby accounting for variation in the morphology of the current beat. In this study, $M$ was empirically set to 60 .

The derived respiratory signal is computed from the QRS complexes $y_{i}(n)$ obtained either without or with f-wave suppression:

$$
y_{i}(n)= \begin{cases}x_{i}(n), & \text { w/o suppression, } \\ \hat{x}_{\mathrm{QRS}, i}(n), & \text { with suppression. }\end{cases}
$$

\section{B. Derived respiratory signals}

1) QRS loop rotation angle $d_{L A}$ : Spatiotemporal alignment between successive, observed QRS loops $\boldsymbol{Y}_{i}$ and a reference QRS loop $\boldsymbol{Y}_{\mathrm{R}}$ is used to estimate a rotation angle reflecting respiratory information [16], see also [28]. For two leads, considered in this study, the reference loop $\boldsymbol{Y}_{\mathrm{R}}$ contains a twolead reference QRS complex and the $i$ :th loop $\boldsymbol{Y}_{i}$ contains the QRS complexes $y_{i}(n)$ of two leads. The loop $\boldsymbol{Y}_{i}$ is assumed to be a version of $\boldsymbol{Y}_{\mathrm{R}}$ but rotated, scaled, and shifted in time. For each beat, the estimated rotation matrix $\hat{\boldsymbol{Q}}_{i}(2 \times 2)$ is used to extract an angle which forms the EDR signal $d_{\mathrm{LA}}(i)$ :

$$
d_{\mathrm{LA}}(i)=\arcsin \left(\hat{\boldsymbol{Q}}_{i}(2,1)\right) .
$$

2) $\boldsymbol{R}$-wave angle $d_{R A}$ : The R-wave angle reflects respiratory information in individual leads. First, using the least squares technique, a straight line is fitted to each side of the QRS complex in $8 \mathrm{~ms}$ intervals centered at the points of maximum absolute slope, see [22] for details. The R-wave angle is defined as the angle formed by two straight lines fitted to the up- and downslope of the QRS complex:

$$
d_{\mathrm{RA}}(i)=\arctan \left(\left|\frac{\mathcal{I}_{\mathrm{US}, i}-\mathcal{I}_{\mathrm{DS}, i}}{1+\mathcal{I}_{\mathrm{US}, i} \mathcal{I}_{\mathrm{DS}, i}}\right|\right)
$$

where $\mathcal{I}_{\mathrm{US}, i}$ and $\mathcal{I}_{\mathrm{DS}, i}$ denote the up- and downslope of the two fitted lines, respectively.

3) Slope range $d_{S R}$ : The slope range is a novel parameter which reflects respiratory information in individual leads. It is defined by the difference between the maximum up-slope and minimum down-slope values within the QRS interval, irrespectively of their relative time occurrence:

$$
d_{\mathrm{SR}}(i)=\max _{n}\left\{y_{i}^{\prime}(n)\right\}-\min _{n}\left\{y_{i}^{\prime}(n)\right\},
$$

where $y_{i}^{\prime}(n)=y_{i}(n)-y_{i}(n-1)$. This definition of the slopes avoids the line fitting used in Sec. II-B2.

The three EDR signals are illustrated in Fig. 1. 

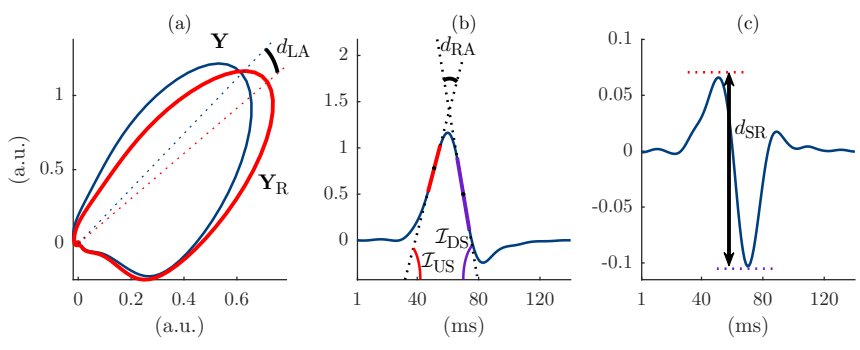

Fig. 1. Derived respiratory signals. (a) rotation angle $d_{\mathrm{LA}}$ of the observed loop $\boldsymbol{Y}$ relative to the reference loop $\boldsymbol{Y}_{\mathrm{R}}$, (b) R-wave angle $d_{\mathrm{RA}}$ between the lines fitted to the upslope (red) and downslope (purple) of the QRS complex $y_{i}(n)$, and (c) slope range $d_{\mathrm{SR}}$ between the absolute maximum (red) and minimum (purple) of the first derivative $y_{i}^{\prime}(n)$ of the QRS complex.

\section{Outlier rejection and resampling}

Outliers in an EDR signal are rejected using a standard deviation (SD)-based rule [16]; the threshold is defined as 5 times the running SD. Since an EDR signal is irregularly sampled, cubic spline interpolation is performed followed by resampling at $F_{s}=4 \mathrm{~Hz}$, where gaps longer than $2 \mathrm{~s}$ are excluded from rate estimation. The resampled EDR signal is indexed with $n$ instead of $i$ and f-wave suppression is indicated with an "s", i.e., $d_{\mathrm{LA}, s}(n), d_{\mathrm{RA}, s}(n)$, and $d_{\mathrm{SR}, s}(n)$.

\section{Respiratory rate estimation}

An estimate $\hat{f}_{r}(k)$ of the respiratory rate is obtained from a smoothed version of the running power spectrum $S_{k, l}(f)$ of the EDR signal, where $k$ and $l$ index the running interval and the analyzed lead ${ }^{1}$, respectively. A block diagram of the respiratory rate estimation is shown in Fig. 2.

Each power spectrum is obtained using the Welch method and computed every $\Delta T \mathrm{~s}$ in intervals of length $T_{s} \mathrm{~s}$, divided into subintervals of length $T_{m}$ s with $50 \%$ overlap [16]. Before computing the averaged power spectrum of the Welch method, the power spectrum of each subinterval is normalized with respect to power to reduce the influence of spurious spectral peaks due to artifacts and to compensate for leadto-lead differences in signal power. The power at frequencies exceeding half the mean heart rate is set to zero.

Assuming that respiration is stationary in an interval of $T_{s} \mathrm{~s}$, a smoothed version of $\hat{f}_{r}(k)$ is obtained by

$$
\bar{f}_{r}(k)=\beta \bar{f}_{r}(k-1)+(1-\beta) \hat{f}_{r}(k),
$$

where $\beta$ denotes the forgetting factor; the details on how to obtain $\hat{f}_{r}(k)$ are presented below.

1) Spectral peakedness: A measure of spectral concentration ("peakedness") is introduced to exclude flat spectra and spectra with low power around $\bar{f}_{r}(k-1)$, defined by

$$
P_{k, l}=\frac{\int_{\boldsymbol{\Omega}_{p}(k)} S_{k, l}(f) d f}{\int_{\boldsymbol{\Omega}(k)} S_{k, l}(f) d f} \cdot 100 \%,
$$

\footnotetext{
${ }^{1}$ Note that $l$ refers to the EDR signal(s), not necessarily to the recorded leads. For two leads, two EDR signals can be derived for $d_{\mathrm{RA}}$, and $d_{\mathrm{SR}}$, while only one EDR signal for $d_{\mathrm{LA}}$.
}

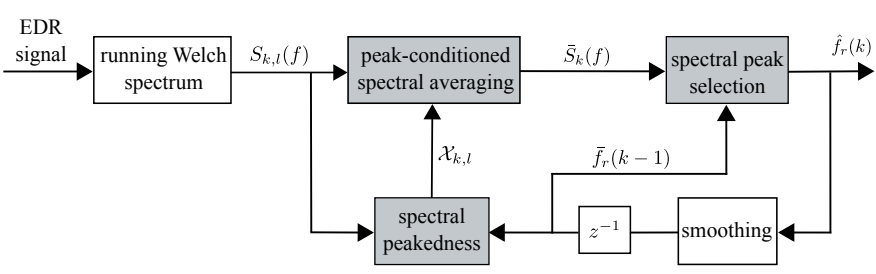

Fig. 2. Block diagram of respiratory rate estimation. The intermediate steps include computation of the running Welch spectrum, the peakedness measure, the peak-conditioned averaging, and spectral peak selection in the averaged spectrum.

where both $\Omega_{p}(k)$ and $\boldsymbol{\Omega}(k)$ are centered around $\bar{f}_{r}(k-1)$ such that:

$$
\begin{aligned}
\boldsymbol{\Omega}_{p}(k) & =\left[\bar{f}_{r}(k-1)-\delta_{p}, \bar{f}_{r}(k-1)+\delta_{p}\right], \\
\boldsymbol{\Omega}(k) & =\left[\bar{f}_{r}(k-1)-\delta, \bar{f}_{r}(k-1)+\delta\right],
\end{aligned}
$$

where $\boldsymbol{\Omega}_{p}(k)$ is contained in $\boldsymbol{\Omega}(k)\left(\delta_{p}<\delta\right)$. Figure 3 shows examples of EDR spectra $S_{k, l}(f)$ with different percentages of power concentration $P_{k, l}$ around $\bar{f}_{r}(k-1)$.

The width parameter $\delta$ should be selected according to the half-width of the expected respiratory band. It should be noted that while $\Omega_{p}(k)$ was centered around the local maximum inside $\boldsymbol{\Omega}(k)$ closest to $\bar{f}_{r}(k)$ in [22], $\boldsymbol{\Omega}_{p}(k)$ is centered around $\bar{f}_{r}(k)$ in this study. A search for the local maxima is avoided since the position of local maxima is highly variable across spectra when f-waves are present.

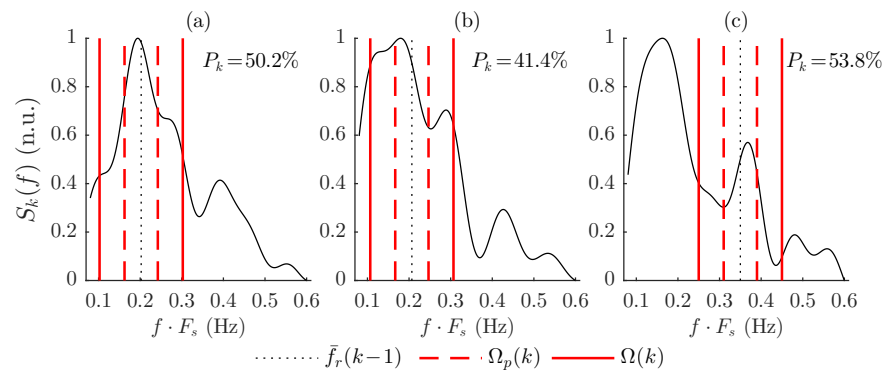

Fig. 3. Examples of spectral peakedness computed using (10). EDR spectrum having a large percentage of its power in $\Omega(k)$ mainly concentrated (a) within $\boldsymbol{\Omega}_{p}(k)$, (b) outside $\boldsymbol{\Omega}_{p}(k)$, (c) within $\boldsymbol{\Omega}_{p}(k)$ although the peak in $\boldsymbol{\Omega}(k)$ is not the absolute maximum of $S_{k}(f)$. For simplicity, the dependence of $l$ in $S_{k, l}(f)$ and $P_{k, l}$ is omitted.

2) Peak-conditioned spectral averaging: The variance reduction provided by the Welch method is complemented with peak-conditioned spectral averaging of $K$ successive running spectra $S_{k, l}(f)$ to further reduce the variance, and thus enhances the frequency component corresponding to the respiratory rate [16]. In addition, averaging is performed across available leads. The spectrum used for respiratory rate estimation is given by

$$
\bar{S}_{k}(f)=\sum_{l} \sum_{o=0}^{K-1} \mathcal{X}_{k-o, l} \cdot S_{k-o, l}(f),
$$

where $\mathcal{X}_{k, l}$ is a binary variable indicating the peakedness of $S_{k, l}(f)$, set to 1 when $P_{k, l}$ exceeds the threshold $\xi$ :

$$
\mathcal{X}_{k, l}= \begin{cases}1, & P_{k, l} \geq \xi_{p} \wedge A_{k, l} \geq \xi_{a}, \\ 0, & \text { otherwise },\end{cases}
$$



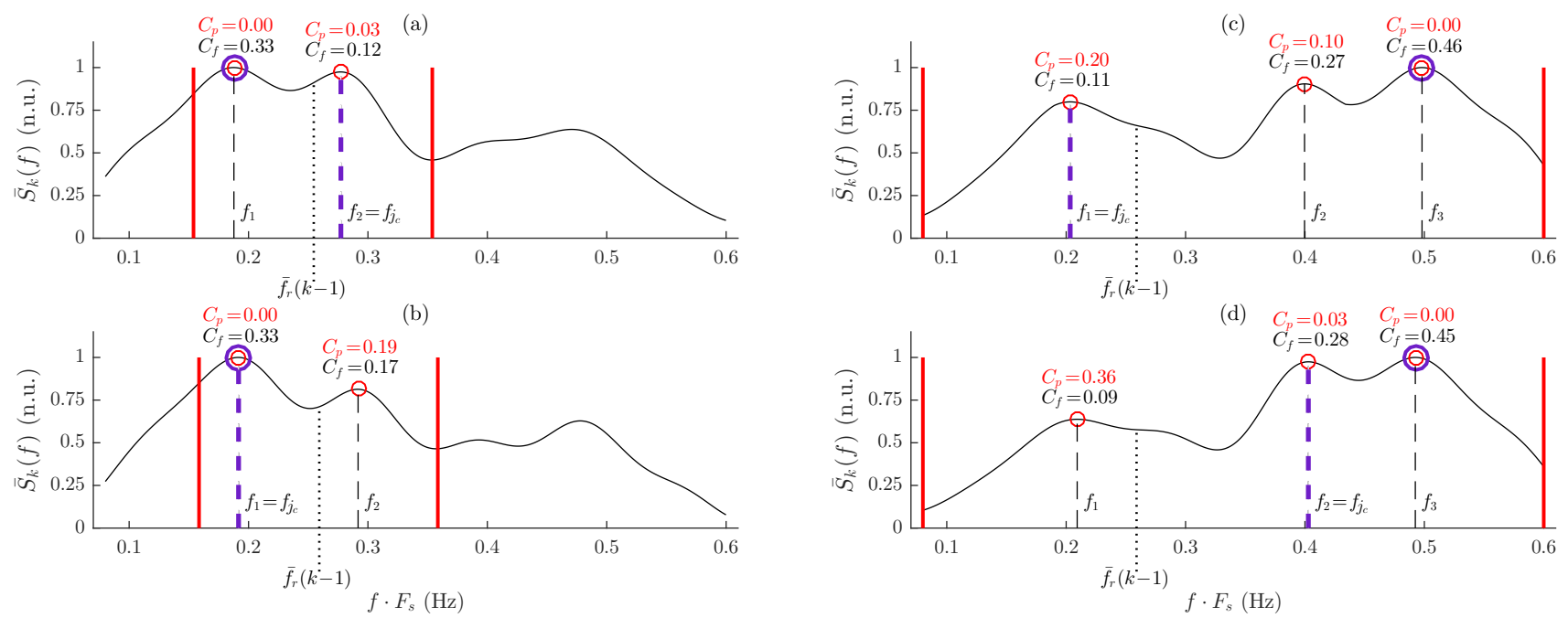

Fig. 4. Cost assignment in respiratory rate estimation and the reinitialization of $\bar{f}_{r}(k)$. (a)-(b) Costs used in peak search within $\boldsymbol{\Omega}(k)$ (red solid lines). (c)-(d) Costs used in peak search within $\boldsymbol{\Omega}_{r}$ (red solid lines). The $\bar{S}_{k, \mathrm{MAX}}$ is marked with a purple circle while the estimated respiratory rate with a purple line. The dependence of $j$ or/and $k$ in $f_{j_{c}}(k), C_{p}(k, j)$, and $C_{f}(k, j)$ is for simplicity omitted.

where

$$
A_{k, l}=\frac{\max _{f \in \boldsymbol{\Omega}(k)}\left\{S_{k, l}(f)\right\}}{\max _{f \in \boldsymbol{\Omega}_{r}}\left\{S_{k, l}(f)\right\}} \cdot 100 \% .
$$

As shown in Fig. 3(c), this restriction is necessary since a large $P_{k, l}$ does not ensure that the spectral peak in $\Omega(k)$ is the largest peak across the whole range of respiratory rates. The range $\Omega_{r}$ is fixed and chosen so that it covers the respiratory rates observed in AF patients.

3) Spectral peak selection: The respiratory rate is selected among the $J$ local maxima $f_{1}(k), \ldots, f_{J}(k)$ in $\bar{S}_{k}(f)$ inside $\boldsymbol{\Omega}(k)$, using minimization of a cost function that quantifies, for each spectral peak, the power deviation from the largest peak and the frequency deviation from $\bar{f}_{r}(k)$. Assuming that the respiratory rate in the $k$ :th interval corresponds to a peak whose power is similar to the global maximum $\bar{S}_{k, \mathrm{MAX}}$, defined by

$$
\bar{S}_{k, \text { MAX }}=\max _{j}\left\{\bar{S}_{k}\left(f_{j}(k)\right)\right\}, j=1, \ldots, J,
$$

and near $\bar{f}_{r}(k-1)$, the power deviation from $\bar{S}_{k, \operatorname{MAX}}$ is penalized by the cost $C_{p}(k, j)$ and the deviation from $\bar{f}_{r}(k-1)$ by $C_{f}(k, j)$ :

$$
\begin{aligned}
C_{p}(k, j) & =1-\bar{S}_{k}\left(f_{j}(k)\right) / \bar{S}_{k, \mathrm{MAX}}, \\
C_{f}(k, j) & =\left|f_{j}(k)-\bar{f}_{r}(k-1)\right| / 2 \delta, \\
j_{c}(k) & =\arg \min _{j}\left\{a_{p} C_{p}(k, j)+a_{f} C_{f}(k, j)\right\},
\end{aligned}
$$

where $a_{p}$ and $a_{f}$ are cost weights. The desired estimate of the respiratory rate is given by

$$
\hat{f}_{r}(k)=f_{j_{c}(k)} \equiv f_{j_{c}}(k) .
$$

The cost assignment and the reinitialization of $\bar{f}_{r}(k)$ (see Appendix for details) for different peak configurations are illustrated in Fig. 4. For the computation of $\hat{f}_{r}(k)$ (Figs. 4 (a)(b)), the cost evaluation is performed for all peaks inside $\boldsymbol{\Omega}(k)$, while, for the reinitialization of $\bar{f}_{r}(k)$ (Figs. 4 (c)-(d)), the cost evaluation is performed inside $\boldsymbol{\Omega}_{r}$. As observed from Fig. 4, rate estimation does not always correspond to the largest peak or the peak closest to $\bar{f}_{r}(k-1)$, but it combines the information of both peak.

The following parameter values are used when evaluating the performance [22]: $K=5, \Delta T=5 \mathrm{~s}, T_{m}=12 \mathrm{~s}, T_{s}=42 \mathrm{~s}$, $\delta=0.1 / F_{s}$. The parameters $\delta_{p}=0.4 \delta, \beta=0.8, \xi_{p}=45 \%$, $\xi_{a}=85 \%, \boldsymbol{\Omega}_{r}=[0.08,0.6] / F_{s}$, and $a_{p}=a_{f}=1$ are set empirically.

\section{Data Sets}

\section{A. Real data}

Estimation performance is evaluated using a database containing recordings from 30 patients with persistent $\mathrm{AF}(69 \pm 15$ years, 17 females) acquired at the Department of Cardiology at San Paolo Hospital in Milan, Italy. The patients underwent electrical cardioversion after having had an AF episode lasting longer than 7 days. The morning before electrical cardioversion, recordings were acquired at rest supine position about $15 \mathrm{~min}$. The ECG was recorded using two non-orthogonal leads $\left(F_{s, \mathrm{ECG}}=1000 \mathrm{~Hz}\right)$ and the reference respiratory signal using a belt sampled at $50 \mathrm{~Hz}$, both signals acquired with a Task Force $\AA$ Monitor (CNSystem; Graz, Austria) recording system.

\section{B. Simulated data}

To shed light on performance, a model for simulating 12-lead ECG signals in AF is employed [30]. Briefly, the signals are generated by simulating QRST complexes whose morphological variability accounts for respiratory influence (angular variation around each lead), real, extracted f-waves, and various types of noise, see Appendix for further details. The SNR between the QRS complex and the f-waves is defined by

$$
\mathrm{SNR}=20 \cdot \log _{10}\left(A_{\mathrm{QRS}} / A_{f}\right),
$$


(a)

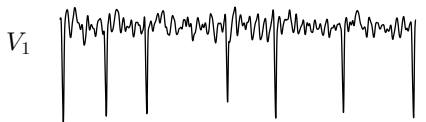

(e)

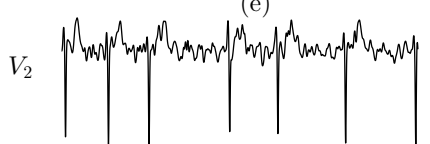

(i)

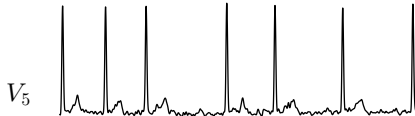

(b)

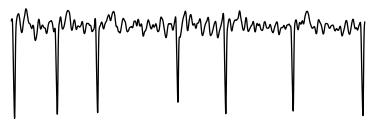

(f)

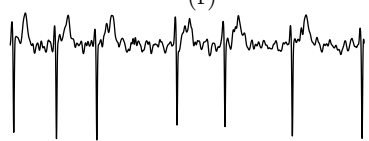

(j)

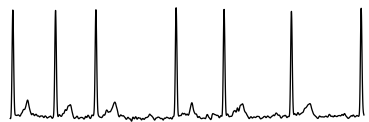

(c)

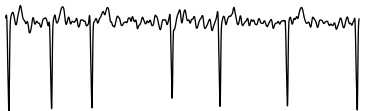

(g)

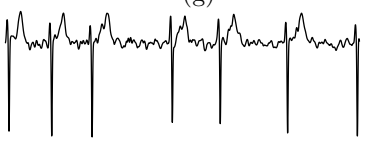

(k)

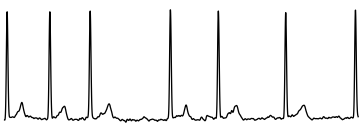

(d)

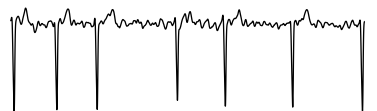

(h)

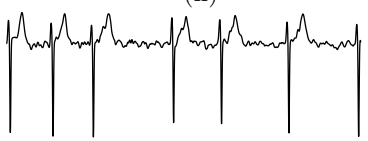

(1)

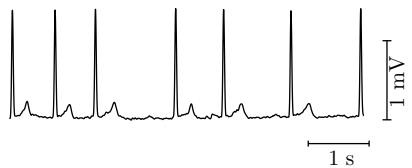

Fig. 5. Simulated ECGs for $F_{r}=0.15 \mathrm{~Hz}$ in lead (a)-(d) $V_{1}$. (e)-(h) $V_{2}$, and (i)-(l) $V_{5}$. In $V_{1}$, the SNR increases from 12 to $21 \mathrm{~dB}$ in steps of $3 \mathrm{~dB}$ (left to right). Note that a larger f-wave amplitude implies a lower SNR.

where $A_{\mathrm{QRS}}$ is the peak-to-peak amplitude of the ensemble averaged $\mathrm{QRS}$ and $A_{f}$ is the peak-to-peak f-wave amplitude computed as the mean of the difference between the upper and the lower envelope of the f-wave signal [31]. Using (21), a lower SNR implies a larger f-wave amplitude. For multi-lead ECGs, the SNR is given by the lead with the lowest SNR. The minimum and maximum SNRs across leads are denoted $\mathrm{SNR}_{\mathrm{MIN}}$ and $\mathrm{SNR}_{\mathrm{MAX}}$, respectively.

\section{Performance Evaluation Setup}

The reference respiratory rate $f_{r}(k)$ is obtained from the reference respiratory signal by applying the same approach as that used in the EDR signals. To evaluate performance, the mean $\mu$ and SD $\sigma$ of the absolute error $\Delta f(k)$ between $f_{r}(k)$ and $\hat{f}_{r}(k)$ and the relative error $\Delta f(k) / f_{r}(k)$ are considered, provided that both $f_{r}(k)$ and $\hat{f}_{r}(k)$ are available. The time during which $\hat{f}_{r}(k)$ cannot be estimated is denoted $\mathcal{T}$ and is expressed as a percentage of the overall length of the analyzed signal.

When real ECGs are analyzed, the only method previously applied to derive respiratory information in AF patients is studied for comparative purposes [24]. The method, namely QRS area $d_{\mathrm{A}}(n)$, is computed using the trapezoidal method [25].

Five-minute simulated ECGs are used for estimating the respiratory rate at different SNRs $(12,15,18$, and $21 \mathrm{~dB})$. Two different lead combinations are investigated for simulation, namely $\left(V_{1}, V_{2}\right)$ and $\left(V_{1}, V_{5}\right)$. For a maximum respiratory induced angular variation $\zeta_{r}=5^{\circ}$, three fixed respiratory rates $F_{r}(0.15,0.25$, and $0.35 \mathrm{~Hz})$ are chosen for inducing beatto-beat changes similar to the respiratory patterns observed in subjects at rest supine position. The peak-to-peak f-wave amplitude in the analyzed leads is presented in Table I. Figure 5 illustrates simulated ECGs at different SNRs. For each combination of $F_{r}$ and SNR, 200 different simulated signals are analyzed and the gross median error metrics $(\bar{\mu}, \bar{\sigma})$ of rate estimation are computed.
TABLE I

PEAK-TO-PEAK AMPLITUdE $A_{f}($ MEAN \pm STD $)$ OF THE $f$-WAVES IN SIMULATED SIGNALS

\begin{tabular}{c|r|r|r|}
\cline { 2 - 4 } & \multicolumn{3}{|c|}{$A_{f}(\mu V)$} \\
\hline SNR & \multicolumn{1}{c|}{$V_{1}$} & \multicolumn{1}{c|}{$V_{2}$} & $V_{5}$ \\
\hline 12 & $206 \pm 60$ & $160 \pm 68$ & $45 \pm 24$ \\
15 & $146 \pm 42$ & $113 \pm 48$ & $32 \pm 17$ \\
18 & $103 \pm 30$ & $80 \pm 34$ & $23 \pm 12$ \\
21 & $73 \pm 21$ & $56 \pm 24$ & $16 \pm 8$ \\
\hline \hline
\end{tabular}

\section{Results}

\section{A. Simulated Data}

Figure 6 illustrates respiratory rate estimation on simulated ECGs: f-wave suppression together with $d_{\mathrm{LA}}(n)$ results in cleaner time-frequency (TF) spectra since spurious components in $d_{\mathrm{LA}, s}(n)$ are suppressed (Figs. 6(a)-(b)). A similar TF pattern is achieved using $d_{\mathrm{RA}}(n)$ (Fig. 6(c)) and $d_{\mathrm{SR}}(n)$ (Fig. 6(e)) without f-wave suppression. On the contrary, the extraction of respiratory information after f-wave suppression does not improve performance (Figs. 6(d)-(f)) since the EDR signal, in certain intervals, is almost completely suppressed.

Figure 7 shows the results for the lead combinations $\left(V_{1}, V_{2}\right)$ and $\left(V_{1}, V_{5}\right)$. It is obvious that $\mathrm{f}$-wave suppression deteriorates the performance of $d_{\mathrm{RA}}(n)$ and $d_{\mathrm{SR}}(n)$, while it significantly improves the performance of $d_{\mathrm{LA}}(n)$. For all considered respiratory rates, the median errors $(\bar{\mu}, \bar{\sigma}) \mathrm{Hz}$ across all respiratory rates are below $0.01 \pm 0.01 \mathrm{~Hz}$ for $\mathrm{SNR}>12 \mathrm{~dB}$ without $\mathrm{f}$ wave suppression for SR and RA, while f-wave suppression is required for LA to achieve this particular limit. The median of the percentage of time $\overline{\mathcal{T}}$ during which the respiratory rate could not be estimated was below $10 \%$ in all cases.

Regarding lead combinations, the performance does not improve when $V_{5}$, having the lowest $A_{f}$ (cf. Table I), is analyzed instead of $V_{2}$. The SNR $\mathrm{SAX}_{\mathrm{MAX}}$ was found to be $3 \pm 2 \mathrm{~dB}$ higher than $\operatorname{SNR}_{\mathrm{MIN}}$ for $\left(V_{1}, V_{2}\right)$, while it was $17 \pm 4 \mathrm{~dB}$ for $\left(V_{1}, V_{5}\right)$. The QRS amplitude $A_{\mathrm{QRS}}$ is equal to $872 \pm 208 \mu \mathrm{V}$, $908 \pm 357 \mu \mathrm{V}, 1195 \pm 345 \mu \mathrm{V}$ in $V_{1}, V_{2}$, and $V_{5}$, respectively. 

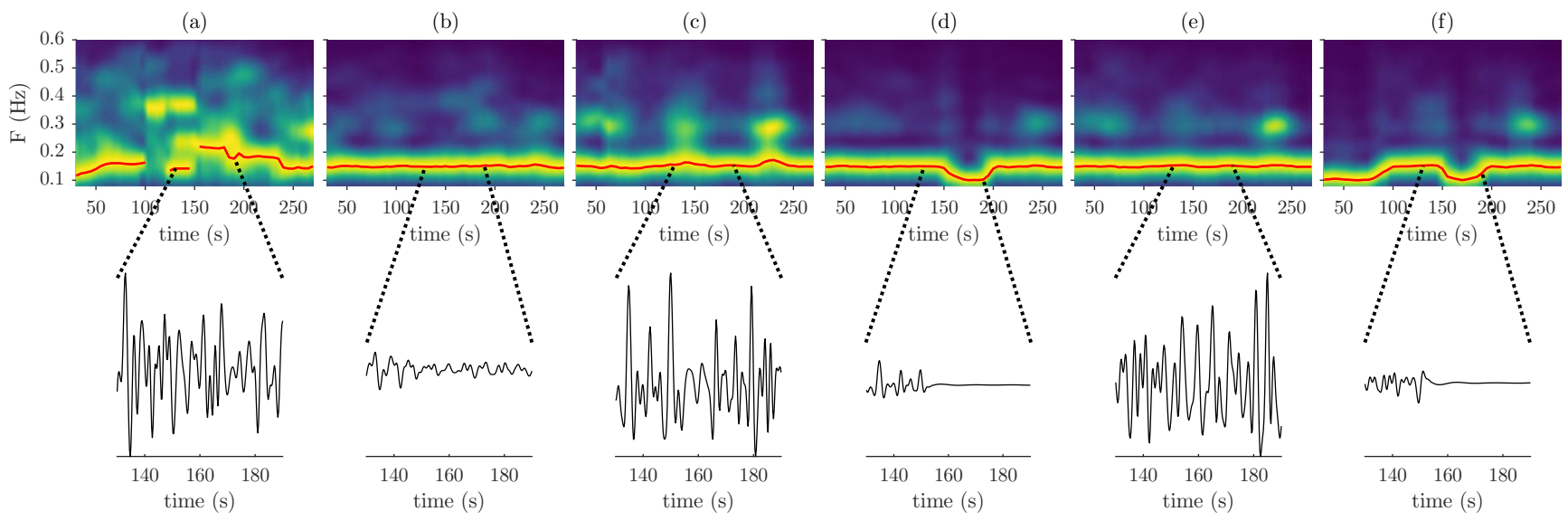

Fig. 6. Respiratory rate estimation in simulated ECGs for $F_{r}=0.15 \mathrm{~Hz}, \mathrm{SNR}=12 \mathrm{~dB}$ and $\left(V_{1}, V_{2}\right)$. Time-frequency spectrum obtained by (a) $d_{\mathrm{LA}}(n)$, (b) $d_{\mathrm{LA}, s}(n)$, (c) $d_{\mathrm{RA}}(n)$, (d) $d_{\mathrm{RA}, s}(n)$, (e) $d_{\mathrm{SR}}(n)$, and (f) $d_{\mathrm{SR}, s}(n)$. The estimated respiratory rate is displayed with a red line. An excerpt of the EDR signal is displayed below each time-frequency spectrum. All EDR signals, with and without f-wave suppression, are plotted with the same amplitude scale.

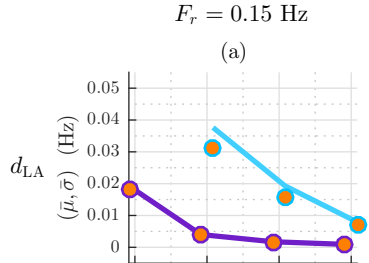

(b)

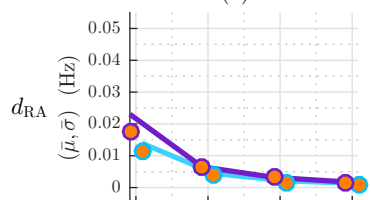

(c)
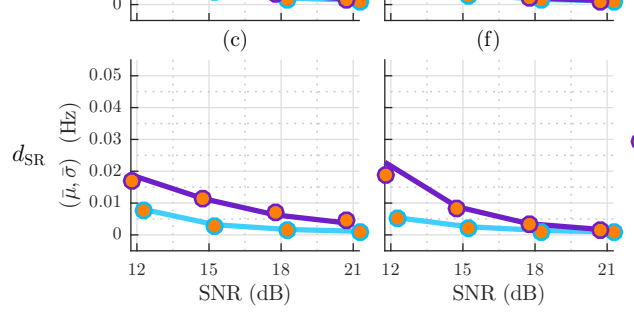

(d)

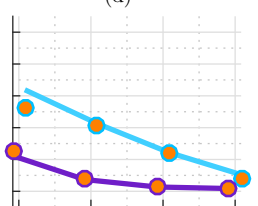

(e)

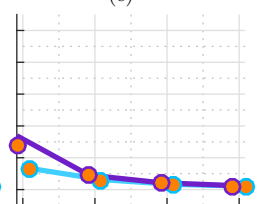

$\left(V_{1}, V_{2}\right)$

$$
F_{r}=0.15 \mathrm{~Hz}
$$

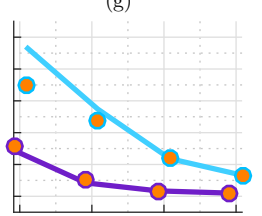

(h)

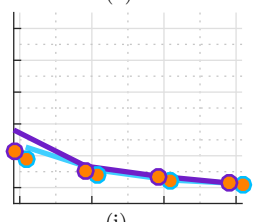

(i)

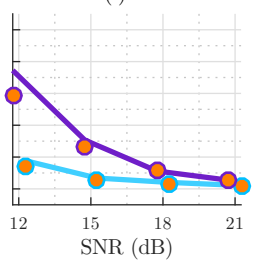

SNR $(\mathrm{dB})$ (j)

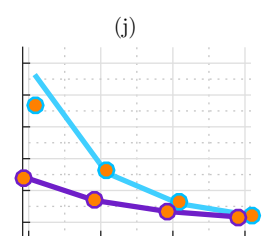

(k)

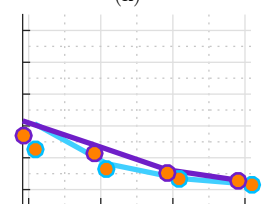

(1)

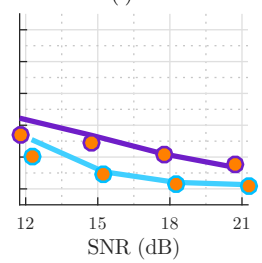

SNR (dB)
$F_{r}=0.25 \mathrm{~Hz}$

(m)

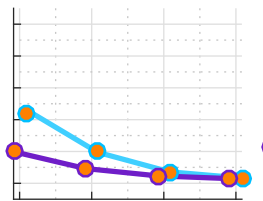

(n)

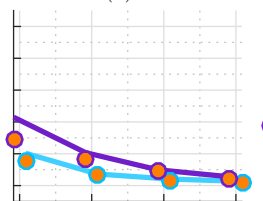

(o)

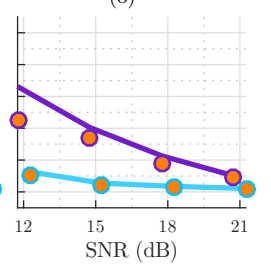

$\left(V_{1}, V_{5}\right)$
$F_{r}=0.35 \mathrm{~Hz}$

(p)

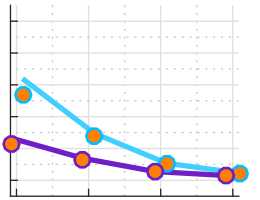

(q)

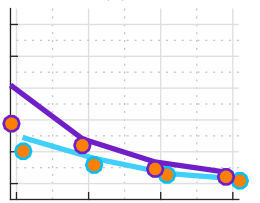

(r)

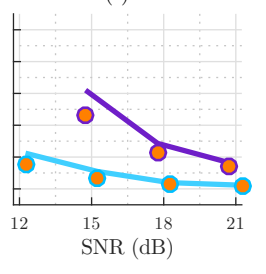

NR (dB)

Fig. 7. Performance in simulated ECGs with different respiratory rates. The median errors for leads (a)-(i) $V_{1}, V_{2}$ and (j)-(r) $V_{1}, V_{5}$. The top row presents to the performance of $d_{\mathrm{LA}}(n)$, and so on. The errors obtained without and with f-wave suppression are marked in blue and purple, respectively. The error metrics $\bar{\mu}$ and $\bar{\sigma}$ are denoted with lines and circles, respectively. Pairs $(\bar{\mu}, \bar{\sigma})$ with errors higher than $0.05 \mathrm{~Hz}$ are not shown. The error metrics for the methods with and without f-wave suppression are slightly displaced horizontally to become discernible. Note that a larger f-wave amplitude implies a lower SNR.

Hence, large differences between $\mathrm{SNR}_{\mathrm{MIN}}$ and $\mathrm{SNR}_{\mathrm{MAX}}$ for $\left(V_{1}, V_{5}\right)$, are associated with low $\mathrm{f}$-wave amplitude and large QRS amplitude.

\section{B. Real data}

The percentage of noisy beats excluded from the analysis is $5 \% \pm 7 \%$, while $\mathrm{SNR}_{\mathrm{MIN}}$ and $\mathrm{SNR}_{\mathrm{MAX}}$ are $21 \pm 7 \mathrm{~dB}$ and $26 \pm 3 \mathrm{~dB}$, respectively. The rates extracted from the reference respiration signal are characterized by $0.259 \pm 0.083 \mathrm{~Hz}$.

Figure 8 shows that of the four EDR signals $d_{\mathrm{A}}(n)$ performs inferior, regardless of the rate estimation technique. The rate estimation technique proposed in [16] yield larger errors than do the present technique and the one in [22].
While the latter two techniques have similar performance, the present rate estimation technique achieves the lowest error when $d_{\mathrm{SR}}(n)$ is used, resulting in a median error of $0.015 \pm 0.021 \mathrm{~Hz}(9.5 \pm 10.2 \%)$, as well as improves significantly the performance of $d_{\mathrm{A}, s}(n)$ resulting in a median error of $0.034 \pm 0.033 \mathrm{~Hz}(13.5 \pm 17.9 \%)$. The median of $\overline{\mathcal{T}}$ for $d_{\mathrm{A}}(n), d_{\mathrm{A}, s}(n), d_{\mathrm{LA}}(n), d_{\mathrm{LA}, s}(n), d_{\mathrm{RA}}(n)$, and $d_{\mathrm{SR}}(n)$ is $9.4 \%, 3.4 \%, 11.0 \%, 5.3 \%, 3.0 \%$, and $1.9 \%$, respectively. Thus, $d_{\mathrm{SR}}(n)$ is the least affected by the presence of f-waves.

Figure 9 shows an example of the respiratory rate estimation in real ECGs. Figure 10 shows an example of peak selection using different methods. 


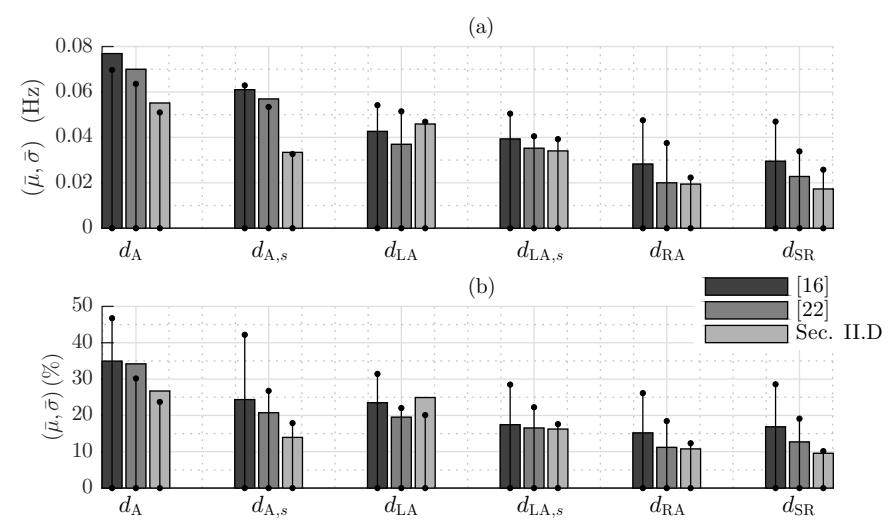

Fig. 8. Respiratory rate estimation in real ECGs using different EDR signals and rate estimation techniques. The (a) absolute error (in $\mathrm{Hz}$ ), and (b) relative error (in \%). The error metrics $\bar{\mu}$ and $\bar{\sigma}$ are denoted with bars and lines, respectively. For each EDR signal, the error metrics of three rate estimation techniques are displayed: the technique in [16] (to the left), [22] (in the middle), and the one presented in Sec. II-D (to the right).

\section{DISCUSSION}

The present paper addresses the lack of methods for estimating the respiratory rate in AF. The estimation is compounded by beat-to-beat morphological variations induced by respiration which are blurred by the presence of f-waves.

The results obtained on simulated ECGs (Fig. 7) show that fwave suppression improves respiratory rate estimation only for $d_{\mathrm{LA}}(n)$. In the same figure, the worse performance of $d_{\mathrm{RA}, s}(n)$ and $d_{\mathrm{SR}, s}(n)$ is largely due to that the weighted averaging operation in (2) leads to smoothing of QRS complexes which in turn attenuates the respiratory information. As demonstrated in Fig. 6, the respiratory information in both $d_{\mathrm{RA}, s}(n)$ and $d_{\mathrm{SR}, s}(n)$ is sometimes completely attenuated because $\hat{\sigma}_{\mathrm{QRS}}^{2}(n)$ is much lower than $\hat{\sigma}_{f}^{2}$, leading to that $\hat{\sigma}_{\mathrm{QRS}}^{2}(n)$ is 0 in (4). For $\hat{\sigma}_{\mathrm{QRS}}^{2}(n)=0$, i.e., the weights in (3) are identical to those of $\mathrm{ABS}$, i.e., $w_{i, m}(n)=1 / M$, the averaged $\mathrm{QRS}$ complexes lose their morphological variability due to respiration since the relationship between $\hat{\sigma}_{\mathrm{QRS}}^{2}(n)$ and $\hat{\sigma}_{f}^{2}$ determines, through (3), the balance between QRS variability preservation $\left(\hat{\sigma}_{f}^{2} \ll \hat{\sigma}_{\mathrm{QRS}}^{2}(n)\right)$ and f-wave suppression $\left(\hat{\sigma}_{f}^{2} \gg \hat{\sigma}_{\mathrm{QRS}}^{2}(n)\right)$. Indeed, the estimation of $\sigma_{f}^{2}$ is a critical part since, at higher heart rates, the TQ intervals may be too short to contain the needed number of samples for reliable estimation. In addition, it is well-known that the f-wave amplitude changes considerably over time, thus questioning the assumption in [26] of a fixed $\sigma_{f}^{2}$.

The suppression of respiratory information appears to depend on the way in which the methods combine the available leads. While $d_{\mathrm{RA}}(n)$ and $d_{\mathrm{SR}}(n)$ consider respiratory information contained in individual leads, $d_{\mathrm{LA}}(n)$ combines spatial information from two leads. This implies that LA will retrieve respiratory information if it is still present or dominant in one of the leads, whereas, for RA and SR, the posterior combination of $\mathrm{TF}$-spectra (one spectrum per lead), where the respiratory information is suppressed, can introduce spurious peaks (Fig. 6(d) and Fig. 6(f)).

The results obtained from real ECGs (Fig. 8) show that the QRS area $d_{\mathrm{A}}(n)$ results in the highest error, thus corroborating the poor performance reported in [24]. More interestingly, using the peakedness proposed in this study, $d_{\mathrm{RA}}(n)$ and $d_{\mathrm{SR}}(n)$ exhibit robust performance without the need for $\mathrm{f}$ wave suppression, yielding median errors of $0.019 \pm 0.025 \mathrm{~Hz}$ $(10.8 \pm 12.3 \%)$ and $0.015 \pm 0.021 \mathrm{~Hz}(9.5 \pm 10.2 \%)$, respectively, which are considerably smaller than those of $d_{\mathrm{LA}}(n)$ and $d_{\mathrm{LA}, s}(n)$, i.e., $0.046 \pm 0.047 \mathrm{~Hz}(24.9 \pm 20.1 \%)$ and $0.034 \pm 0.039 \mathrm{~Hz}(16.2 \pm 17.6 \%)$, respectively. The suppression of f-waves improves the performance of $d_{\mathrm{LA}}(n)$, since variations in the location of the QRS interval, complicating the time alignment between the observed and the reference loop, are reduced. The worse performance of $d_{\mathrm{LA}}(n)$ is mostly attributed to the fact that loop alignment is more difficult to perform in the presence of f-waves, but also to the use of non-orthogonal leads and the way leads are combined.

Since the respiratory rate is known to fluctuate on a shortterm basis, it is of interest to judge whether the estimation error $\Delta f(k)$ is comparable to the variability of $f_{r}(k)$, computed from the reference respiratory signal, and thus acceptable for use in clinical applications. In [16], in stress test recordings, the short-term variability was found to be $0.019 \pm 0.007 \mathrm{~Hz}$ from analysis intervals of 60 seconds. Using the approach in [16], the short-term variability in $f_{r}(k)$ is in the present study found to be $0.012 \pm 0.01 \mathrm{~Hz}$. Since this short-term variability is of the same order of magnitude as the error of the SR method, i.e., $0.015 \pm 0.021 \mathrm{~Hz}$, see Fig. 8, it can be concluded that SR is suitable for use in clinical applications.

The use of simulated ECGs is of great value as it can demonstrate whether f-wave suppression improves the performance since the reference respiratory rate $f_{r}(n)$ can be modified in a controlled manner. This stands in contrast to real ECGs where $f_{r}(n)$ needs to be estimated from the reference respiratory signal. Moreover, the performance can be tested at different SNRs which not only facilitates the interpretation of the results obtained from real ECGs but also provides complementary information. For example, it is shown that the contribution of f-wave suppression is insignificant at high SNRs, whereas, it is essential for LA at low SNRs.

The slope-based methods $d_{\mathrm{RA}}(n)$ and $d_{\mathrm{SR}}(n)$ are less affected by the presence of f-waves since the differencing operation attenuates lower frequencies where most of the fwave power is located. Both SR and RA combine estimates of QRS slopes; the combination can be either linear (8) or nonlinear (7). The use of the first difference is equivalent to use linear fitting with two samples. Computed results evidence that SR obtained either using the first difference or line fitting (8 ms) give equivalent results (not shown). Thus, the slightly worse performance of $d_{\mathrm{RA}}(n)$ relative to $d_{\mathrm{SR}}(n)$ (Fig. 8 and Fig. 7) should be a consequence of the different ways the slope estimates are combined in (7) and (8).

The combination of TF-spectra from more than one EDR signal is advantageous when one of the leads is more influenced by respiration than f-waves. The reason is that the peakedness criteria lead to the selection of the TF-spectra with the most dominant frequency component, not necessarily corresponding to the lead with the lowest f-wave amplitude. An ECG lead with large f-waves may be more adequate for respiratory rate estimation if the morphological changes due to respiration dominate over f-waves. Obviously, the peak-to- 
(a)

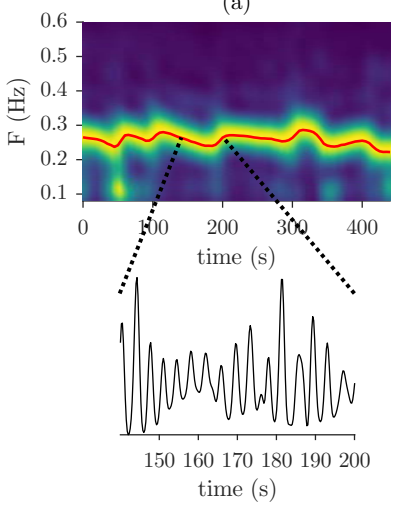

(b)

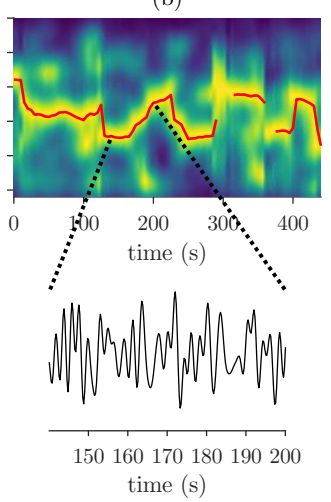

(c)

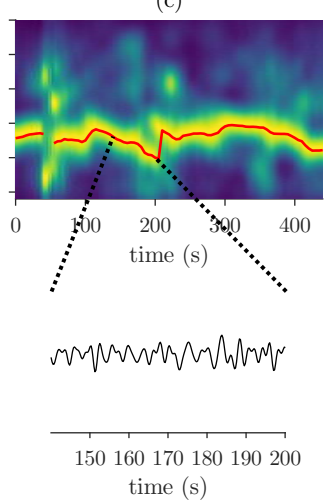

(d)

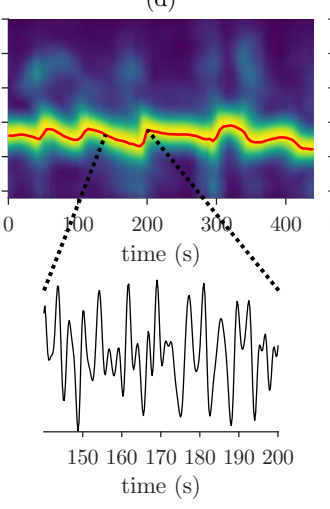

(e)

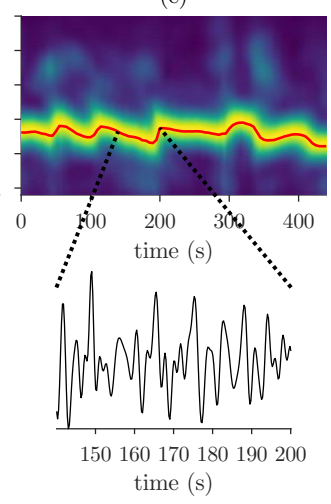

Fig. 9. Respiratory rate estimation in real ECGs. Time-frequency spectrum obtained from (a) the reference respiratory signal, (b) $d_{\mathrm{LA}}(n),(\mathrm{c}) d_{\mathrm{LA}, s}(n)$, (d) $d_{\mathrm{RA}}(n)$, and (e) $d_{\mathrm{SR}}(n)$. The estimated rate is displayed with a red line. An excerpt of the EDR signal is displayed below each TF spectrum.

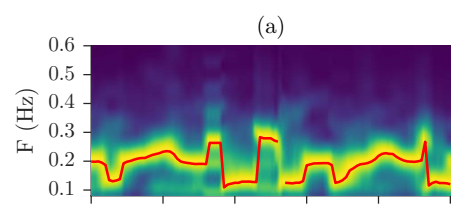

(c)

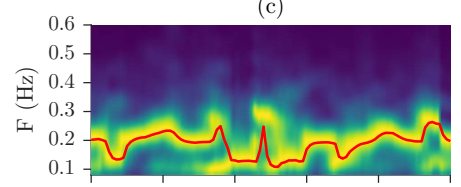

(e)
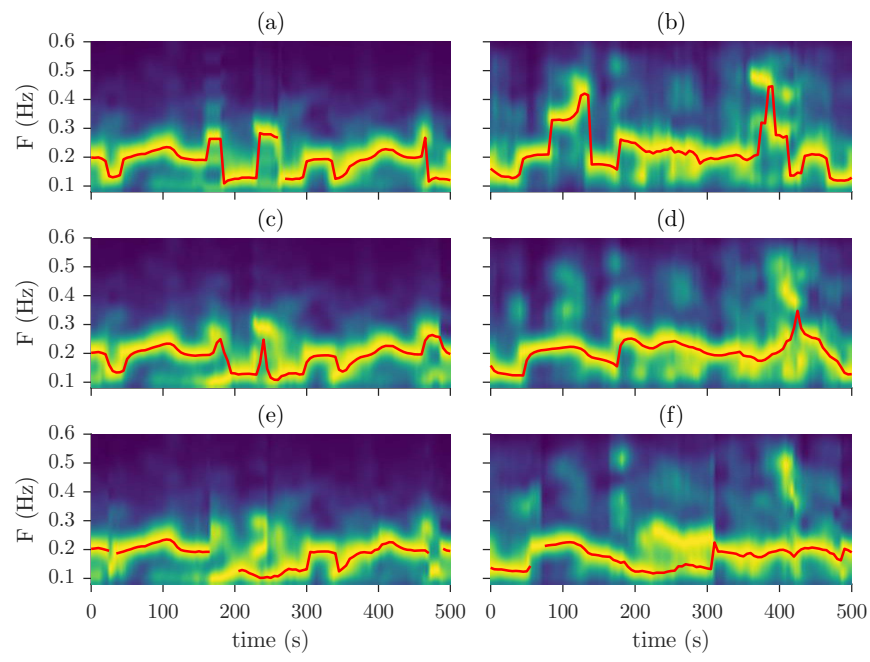

(d)

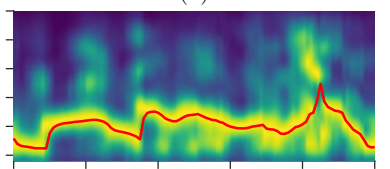

(f)

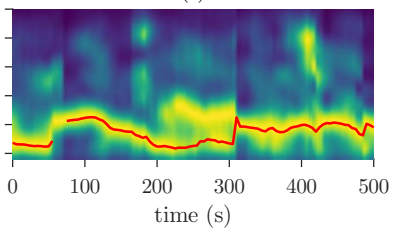

Fig. 10. Respiratory rate estimation in real ECGs using different methods. The left column presents TF spectra obtained from the reference respiratory signal and the right column from the EDR signal $d_{\mathrm{SR}}(n)$, while each row corresponds to the rate estimation procedure in (a)-(b) [16], (c)-(d) [22], (e)-(f) this study. The resulting intra-subject errors are $0.062 \pm 0.063 \mathrm{~Hz}$, $0.040 \pm 0.046 \mathrm{~Hz}$, and $0.016 \pm 0.017 \mathrm{~Hz}$, respectively. The estimated respiratory rate is displayed with a red line.

peak f-wave amplitude is more prominent in $V_{1}$ and $V_{2}$ than in $V_{5}$ (Table I). However, $V_{5}$ is less influenced by respiration than $V_{2}$ since the performance does not improve when analyzing $\left(V_{1}, V_{2}\right)$ instead of $\left(V_{1}, V_{5}\right)$, suggesting that the influence of both respiration and f-waves vary from lead to lead.

In the EDR signals, morphological changes in the QRS complexes due to the f-waves are manifested by additional spectral components complicating the estimation of a timevarying respiratory rate. Moreover, the intrinsic sampling rate of the EDR signal is determined by the heart rate and thus aliasing will occur for components whose frequencies exceed half the mean heart rate. The alias of the first harmonic of the f-waves may lie in the frequency range of respiratory rates and therefore introduce spurious peaks.

To address this problem, a novel definition of peakedness is introduced as well as novel criteria for the selection of a spectral peak defining the respiratory rate. In [16], the respiratory rate is determined by the location of the peak with the largest spectral power, while, in [22], by the location of the peak closest to a smoothed respiratory rate estimate $\bar{f}_{r}(k)$. In this study, respiratory rate estimation is performed through minimization of a cost function which, for each spectral peak, quantifies the power deviation from the largest peak and the frequency deviation from $\bar{f}_{r}(k)$. The peak selection procedures in [16] and [22] are special cases of the proposed method, obtained by either setting $a_{f}=0$ or $a_{p}=0$.

The significance of the peak selection criteria on performance is evaluated on real ECGs. Analyzing $d_{\mathrm{SR}}(n)$, the results in Fig. 8 show that the peakedness measure in (10) achieves the lowest error, while similar errors are obtained for $d_{\mathrm{LA}, s}(n)$ and $d_{\mathrm{RA}}(n)$ using the peakedness measure in [22]. The peakedness measure in [16] yields a larger error than do the other two measures since its design parameters were chosen to account for the dynamics of a stress test. The results suggest that the technique for respiratory rate estimation proposed in this study perform well in the presence of spectral components caused by f-waves. The technique may provide better tracking of respiratory rate in patients with SDB when the spectral components are caused by either amplitude or frequency modulation in the respiratory signal.

This work has focused on evaluating single EDR methodologies with and without f-wave cancellation. Further studies can be done by fusing information from different EDR signals to further improve the accuracy of the respiratory rate estimation, as proposed in [22].

A limitation of the present study is that the performance was studied on only one respiratory rate pattern in simulated signals. Another limitation is that only one f-wave suppression method was investigated. Other methods which better preserve the respiratory information than does signal- and noise-dependent weighted averaging should be investigated. It should also be pointed out that the real ECGs were recorded during rest and therefore further investigations should be undertaken to establish the performance on ECGs recorded in ambulatory conditions. Nonetheless, the results based on simulated ECGs give an idea of performance under such conditions. 


\section{CONCLUSIONS}

Based on real and simulated ECGs, the results show that the slope range method offers the best performance in respiratory rate estimation. Moreover, this method is computationally simple since f-wave suppression is not needed. The R-wave angle method also offers robust performance without needing f-wave suppression, and, therefore, it can be concluded that the slope-based methods are less affected by the presence of f-waves.

\section{APPENDIX}

\section{A. Computation of $\bar{f}_{r}(k)$}

The computation of $\bar{f}_{r}(k)$ involves a number of special cases. At initialization, $\bar{S}_{0}(f)$ is constructed using $2 K$ spectra and $\Omega_{p}$ and $\Omega$ are centered around the global maximum of $\bar{S}_{0}(f)$. Then, $\bar{f}_{r}(0)$ is determined by finding the highest local maximum in $\boldsymbol{\Omega}_{r}$.

If no spectrum is peaked enough, $\bar{S}_{k}(f)$ is not defined; thus, $\hat{f}_{r}(k)$ is not computed and, accordingly, $\bar{f}_{r}(k)=\bar{f}_{r}(k-1)$. If no estimate has been produced for the last $15 \mathrm{~s}$, either due to an abrupt change in $f_{r}(k)$ or incorrect estimation of $f_{r}(k), \bar{f}_{r}(k)$ is reinitialized. The reinitialization is performed by finding the local maximum in $\Omega_{r}$ associated with the minimum cost in (19), leading to

$$
\bar{f}_{r}(k)=f_{j_{c}}(k),
$$

where the bandwidth of the analyzed frequency band in $C_{f}(k, j)$ is based on $\boldsymbol{\Omega}_{r}$.

\section{B. Simulated ECGs}

1) QRST Complexes: The multi-lead ECG model in [32] is used for simulating QRST complexes with different morphologies, see [30] for further details. A synthesized orthogonal three-lead VCG signal $\boldsymbol{u}_{\mathrm{QRS}}(n)(3 \times 1)$ is constructed by concatenating a single QRST complex for each lead $(X, Y, Z)$ until the desired length is attained. The Physionet Long Term Atrial Fibrillation Database is used for creating a set of AF rhythms.

2) $f$-waves: A set of 20 segments with real, multi-lead $\mathrm{f}$ waves $\boldsymbol{u}_{f}(n)(12 \times 1)$ is extracted from a database with 12 lead ECGs acquired from patients clinically diagnosed with persistent AF [33].

3) Noise: A noise component $\boldsymbol{u}_{n}(n)(12 \times 1)$ is composed of two types of noise frequently encountered in ambulatory recordings, muscle noise and electrode movement artifacts extracted from the MIT BIH Noise Stress Test Database. Noise with $20 \mu \mathrm{V}$ RMS is included to better mimic real ECGs [30].

4) Respiratory Influence: In [34], see also [35], it was proposed that the angular variation around each axis (lead $l$ ) is proportional to the amount of air in the lungs during the $p$ :th respiratory cycle. The time-varying angles are modeled by the product of two sigmoidal functions reflecting inhalation and exhalation:

$$
\phi_{l}(n)=\sum_{p=1}^{\infty} \frac{\zeta_{r}}{\zeta_{0}}\left(\frac{1}{1+e^{\lambda_{\mathrm{IN}}(p)\left(n-n_{\mathrm{IN}}(p)\right)}}\right)\left(\frac{1}{1+e^{\lambda_{\mathrm{EX}}(p)\left(n-n_{\mathrm{EX}}(p)\right)}}\right),
$$

$$
\begin{aligned}
\lambda_{\mathrm{IN}}(p) & =-20 \frac{F_{r}(p)}{F_{s, \mathrm{ECG}}}, \\
n_{\mathrm{IN}}(p) & =n_{d}(p)+0.35 \frac{F_{s, \mathrm{ECG}}}{F_{r}(p)} \\
\lambda_{\mathrm{EX}}(p) & =15 \frac{F_{r}(p)}{F_{s, \mathrm{ECG}}}, \\
n_{\mathrm{EX}}(p) & =n_{d}(p)+0.6 \frac{F_{s, \mathrm{ECG}}}{F_{r}(p)}, \\
n_{d}(p) & =\sum_{j=1}^{p} \frac{F_{s, \mathrm{ECG}}}{F_{r}(j)}-\frac{F_{s, \mathrm{ECG}}}{F_{r}(1)},
\end{aligned}
$$

where $1 / \lambda_{\mathrm{IN}}(p)$ and $1 / \lambda_{\mathrm{EX}}(p)$ are the duration of inhalation and exhalation, respectively, $n_{\mathrm{IN}}(p)$ and $n_{\mathrm{EX}}(p)$ are the respective time delays of the sigmoidal functions based on the delay of the previous cycles $n_{d}(p), F_{r}(p)$ is the respiratory rate, and $\zeta_{\mathrm{R}}$ is the maximum angular variation. The maximum value of the product of the two sigmoidal functions, located at the time instant $\frac{\lambda_{\mathrm{IN}}(p) n_{\mathrm{IN}}(p)-\lambda_{\mathrm{EX}}(p) n_{\mathrm{EX}}(p)}{\lambda_{\mathrm{IN}}(p)-\lambda_{\mathrm{EX}}(p)}$ (intersection point), consists in the normalization term $\zeta_{0}$ :

$$
\zeta_{0}=\left(\frac{1}{1+e^{-\frac{n_{\mathrm{IN}}(p)-n_{\mathrm{EX}}(p)}{1 / \lambda_{\mathrm{IN}}(p)-1 / \lambda_{\mathrm{EX}}(p)}}}\right)^{2} \approx 0.8 .
$$

Taking into account that $n_{\mathrm{IN}}(p)$ and $n_{\mathrm{EX}}(p)$, as well as $1 / \lambda_{\mathrm{IN}}(p)$ and $1 / \lambda_{\mathrm{EX}}(p)$, are analogous to $F_{s, \mathrm{ECG}} / F_{r}(p), \zeta_{0}$ becomes fixed and independent of the $p$ :th respiratory cycle.

Finally, the simulated standard 12-lead ECG results from a linear transformation of the three orthogonal leads $X, Y, Z$ [36]:

$$
\boldsymbol{y}(n)=\boldsymbol{D} \boldsymbol{Q}(n) \boldsymbol{u}_{\mathrm{QRS}}(n)+\boldsymbol{u}_{f}(n)+\boldsymbol{u}_{n}(n),
$$

where $\boldsymbol{D}(12 \times 3)$ is the Dower matrix, and $\boldsymbol{Q}(n)$ is the rotation matrix defined as the product of planar rotations with timevarying angles $\phi_{l}(n)$.

\section{REFERENCES}

[1] P. Buch et al., "Reduced lung function and risk of atrial fibrillation in the Copenhagen city heart study," Eur. Respir. J., vol. 21, no. 6, pp. 1012-1016, 2003.

[2] C. Terzano et al., "Atrial fibrillation in the acute, hypercapnic exacerbations of COPD," Eur. Rev. Med. Pharmacol. Sci., vol. 18, no. 19, pp. 2908-17, 2014.

[3] K.F. Rabe et al.,"Global strategy for the diagnosis, management, and prevention of chronic obstructive pulmonary disease: GOLD executive summary," Am. J. Respir. Crit. Care, vol. 176, no. 6, pp. 532-555, 2007.

[4] I. H. Stevenson et al., "Prevalence of sleep disordered breathing in paroxysmal and persistent atrial fibrillation patients with normal left ventricular function," Eur. Heart. J., vol. 29, no. 13, pp. 1662-1669, 2008.

[5] V. Shah. et al., "The association between chronic obstructive pulmonary disease (COPD) and atrial fibrillation: A review," Chron. Obstruct. Pulmon. Dis., vol. 1, no. 2, pp. 1-6, 2016.

[6] C. A. Goudis and D. G. Ketikoglou, "Obstructive sleep and atrial fibrillation: Pathophysiological mechanisms and therapeutic implications," Int. J. Cardiol., vol. 230, pp. 293-300, 2017.

[7] C. Y. Ng et al., "Meta-analysis of obstructive sleep apnea as predictor of atrial fibrillation recurrence after catheter ablation," Am. J. Cardiol., vol. 108 , no. 1, pp. 47-51, 2011.

[8] K. Monahan et al., "Triggering of nocturnal arrhythmias by sleepdisordered breathing events," J. Am. Coll. Cardiol., vol. 54, no. 19, pp. $1797-1804,2009$

[9] J. N. Johnson et al., "Prevalence of early-onset atrial fibrillation in congenital long QT syndrome," Heart Rhythm, vol. 5, no. 5, pp. 704 709, 2008 . 
[10] E. H. Weiss et al., "An optimized method for the estimation of the respiratory rate from electrocardiographic signals: implications for estimating minute ventilation," Am. J. Physiol. Heart Circ. Physiol., vol. 307, no. 3, pp. H437-H447, 2014.

[11] C. Orphanidou, "Derivation of respiration rate from ambulatory ECG and PPG using ensemble empirical mode decomposition: Comparison and fusion," Comput. Biol. Med., vol. 81, pp. 45-54, 2017.

[12] L. V. Højkjær, et al., "Impedance pneumography for long-term monitoring of respiration during sleep in adult males," Clin. Physiol., vol. 4, no. 4, pp. 333-342, 1984.

[13] R. Bailón, L. Sörnmo, and P. Laguna, "ECG-derived respiratory frequency estimation," Advanced methods and tools for ECG data analysis, vol. 1, pp. 215-244, 2006.

[14] P. H. Charlton, et al., "Breathing rate estimation from the electrocardiogram and photoplethysmogram: A review," IEEE Rev. Biomed. Eng., vol. 11, no. 6, pp. 2-20, 2018.

[15] S. Leanderson, P. Laguna, and L. Sörnmo, "Estimation of the respiratory frequency using spatial information in the VCG," Med. Eng. Phys., vol. 25, no. 6, pp. 501-507, 2003.

[16] R. Bailón, L. Sörnmo, and P. Laguna, "A robust method for ECGbased estimation of the respiratory frequency during stress testing," IEEE Trans. Biomed. Eng., vol. 53, no. 7, pp. 1273-1285, 2006.

[17] S. Babaeizadeh et al., "Electrocardiogram-derived respiration in screening of sleep-disordered breathing," J. Electrocardiol., vol. 44, no. 6, pp. 700-706, 2011.

[18] C. Maier et al., "Prospective evaluation of a Holter-ECG derived severity index for screening of sleep disordered breathing," J. Electrocardiol., vol. 49, no. 6, pp. 919-924, 2016.

[19] P. Langley, E. J. Bowers, and A. Murray, "Principal component analysis as a tool for analyzing beat-to-beat changes in ECG features: application to ECG-derived respiration," IEEE Trans. Biomed. Eng., vol. 57, no. 4, pp. 821-829, 2010

[20] D. Widjaja et al., "Application of kernel principal component analysis for single-lead-ECG-derived respiration," IEEE Trans. Biomed. Eng., vol. 59, no. 4, pp. 1169-1176, 2012.

[21] N. Sadr and P. Chazal,"A fast approximation method for principal component analysis applied to ECG derived respiration for OSA detection," Conf. Proc. IEEE Eng. Med. Biol. Soc., pp. 6198-6201, 2016.

[22] J. Lázaro et al., "Electrocardiogram derived respiratory rate from QRS slopes and R-wave angle," Ann. Biomed. Eng., vol. 42, no. 10, pp. 2072 2083, 2014.

[23] D. Widjaja et al., "ECG-derived respiration: Comparison and new measures for respiratory variability," in Proc. Comput. Cardiol., 2010, pp. $149-152$.

[24] V. Pichot et al., "ECG-derived respiration: A promising tool for sleepdisordered breathing diagnosis in chronic heart failure patients," Int. J. Cardiol., vol. 186, pp. 7-9, 2015.

[25] G.B. Moody et al., "Derivation of respiratory signals from multi-lead ECGs," Comput. Cardiol., vol. 12, pp. 113-116, 1985.

[26] H. Dai, S. Jiang, and Y. Li, "Atrial activity extraction from single lead ECG recordings: Evaluation of two novel methods," Comput. Biol. Med., vol. 43, no. 3, pp. 176-183, 2013.

[27] L. Sörnmo et al., "Extraction of f waves," in Atrial Fibrillation from an Engineering Perspective, L. Sörnmo (Ed.), Springer, 2018, ch. 5, pp. 137-220.

[28] L. Sörnmo, "Vectorcardiographic loop alignment and morphologic beatto-beat variability," IEEE Trans. Biomed. Eng., vol. 45, no. 12, pp. 14011413, 1998.

[29] M. Åström et al., "Detection of body position changes using the surface electrocardiogram," Med. Biol. Eng. Comput., vol. 41, no. 2, pp. 164 171, 2003.

[30] A. Petrẻnas et al., "Electrocardiogram modeling during paroxysmal atrial fibrillation: application to the detection of brief episodes," Phys. Meas., vol. 38, no. 2, pp. 2058-2080, 2017.

[31] M. Meo et al., "Spatial variability of the 12-lead surface ECG as a tool for noninvasive prediction of catheter ablation outcome in persistent atrial fibrillation," IEEE Trans. Biomed. Eng., vol. 60, no. 1, pp. 20-27, 2013.

[32] R. Sameni et al., "Multichannel ECG and noise modeling: Application to maternal and fetal ECG signals," J. Adv. Signal Process., vol. 2007, no. 1, pp. 94-94, 2007.

[33] A. Petrènas et al., "An echo state neural network for QRST cancellation during atrial fibrillation," IEEE Trans. Biomed. Eng., vol. 59, no. 10, pp. 2950-2959, 2012

[34] M. Åström et al., "Vectorcardiographic loop alignment and the measurement of morphologic beat-to-beat variability in noisy signals," IEEE Trans. Biomed. Eng., vol. 47, no. 4, pp. 497-506, 2000.
[35] L. Sörnmo et al., "Databases and Simulation," in Atrial Fibrillation from an Engineering Perspective, L. Sörnmo (Ed.), Springer, 2018, ch. 3, pp. 49-72.

[36] G. E. Dower, H. B. Machado, and J. Osborne, "On deriving the electrocardiogram from vectoradiographic leads." Clin. Cardiol., vol. 3, no. 2 , pp. 87-95, 1980 . 